Maurício Leite de Oliveira²

Joaquim Gonçalves Machado Neto ${ }^{3}$

\title{
Segurança na aplicação de agrotóxicos em cultura de batata em regióes montahosas ${ }^{1}$ Safety of pesticides application on potato crop on sloped areas
}

\begin{abstract}
Resumo
O presente estudo objetivou a quantificação das exposições na aplicação de agrotóxicos com o pulverizador convencional usado pelo produtor em cultura de batata em regiões montanhosas; a determinação das regiões do corpo dos trabalhadores mais expostas; a avaliação da eficiência de medidas de proteção individuais e coletivas; e a classificação das condições de trabalho quanto à segurança das recomendações de agrotóxicos registrados para o controle das principais pragas e doenças dessa cultura. Concluiu-se que a maior exposição ocupacional ocorreu na via dérmica; o trabalhador sofreu maior exposição com a utilização do pulverizador do produtor; o Knapik foi a medida de segurança coletiva mais eficiente no controle da exposição do trabalhador e a individual mais eficiente foi a Roupa Unesp; os membros inferiores foram as regiões do corpo mais exposta às caldas; sem a utilização das medidas de segurança, as 54 recomendações de agrotóxico para a essa cultura foram classificadas como inseguras para o trabalhador; todavia, com a utilização do protótipo de barra manual, apenas duas tornaram-se seguras; com o pulverizador Knapik, somente três; nove com o AZR; e dezesseis com a Roupa Unesp.
\end{abstract}

Palavras-chaves: agrotóxicos, exposição ocupacional, risco de intoxicação, batata.

\begin{abstract}
The aim of the present study was a) to quantify exposure in the application of pesticides on potatoes with a conventional sprayer on sloped areas, b) to determine the areas of the body where workers are most exposed, $c$ ) to assess the effectiveness of protective measures both individual and collective, and d) to classify the working conditions with regard to work safety in the recommended use of pesticides registered for this crop. The following results were obtained. The greatest occupational exposure occurred via the dermal route. The worker was found to have the greatest exposure when using a crop sprayer. The Knapik was the most efficient collective safety measure in controlling exposure of the worker, while the Roupa Unesp was the most efficient individual safety measure. The worker's legs were the area of the body most exposed to the spray. Without utilizing any of the safety measures evaluated, all of the 54 recommendations for the application of pesticides on this crops would be classified as unsafe for the worker. However, when the sprayer prototype with manual boom was used, only two turned out to be safe, whereas only three with the Knapik sprayer, nine with the AZR, and 16 with the Roupa Unesp.
\end{abstract}

Keywords: pesticide, occupational exposure, intoxication hazard, potato. 


\section{Introdução}

O desenvolvimento dos pulverizadores agrícolas, nos últimos anos, possibilitou maior eficiência da pulverização, diminuição do volume aplicado e aumento na segurança dos operadores. Porém, tal desenvolvimento não proporcionou mudanças no método de pulverização utilizado por pequenos e médios agricultores da cultura de batata em regiões montanhosas. Nessa cultura, o pulverizador semi-estacionário acoplado à mangueira arrastada no interior da cultura é o mais utilizado. Essa condição de trabalho de pulverização de agrotóxicos proporciona alto nível de exposição ocupacional e risco de intoxicação dos trabalhadores devido principalmente à pequena distância entre os bicos pulverizadores e o operador e ao elevado volume de calda de agrotóxico aplicado por hectare. A grande quantidade de agrotóxicos aplicados também aumenta a possibilidade de intoxicações ocupacionais, pois a cultura da batata destacou-se em sétimo lugar em vendas de agrotóxicos, atingindo 61.665 milhões de dólares em 2000 (SINDAG, 2004).

Como medida de segurança no trabalho com agrotóxicos, geralmente se recomenda apenas o uso de equipamentos de proteção individual (EPIs), que controlam as exposições dérmicas e respiratórias proporcionadas por condições de trabalho específi-

\section{Material e Métodos}

As exposições dos aplicadores de agrotóxicos com o pulverizador convencional do produtor, com um protótipo da barra manual e com o pulverizador Knapik foram avaliadas em pulverizações na cultura da batata cultivada em região montanhosa, onde a operação de pulverização é realizada de maneira semi-mecanizada. A cultura estava plantada com espaçamento de 0,75 $\mathrm{m}$ nas linhas e com as plantas cobrindo todo o solo.

No pulverizador convencional do produtor, utiliza-se de uma vara de bambu de 7,5 m de comprimento, como barra de pulverização, conectada a um conjunto de pulverizador Jacto Condor M-12, com uma mangueira de $12,7 \mathrm{~mm}$ e $400 \mathrm{~m}$ de comprimento. O pulverizador, acoplado a um trator MF-275 acelerado a $1.200 \mathrm{rpm}$, ficou estacionado no carreador contíguo à área pulverizada. cas. Habitualmente, esses equipamentos são erroneamente recomendados com base apenas na classe toxicológica dos agrotóxicos e não na exposição ocupacional que as condições de trabalho propiciam e na sua distribuição pelo corpo do trabalhador.

Para gerenciar-se a segurança das condições de trabalho com agrotóxicos, o conhecimento das exposições dérmica e respiratória potenciais, das características tóxicas dos agrotóxicos e da eficiência das medidas de proteção é de grande importância para a seleção e a adoção das medidas de segurança mais efetivas, confortáveis, econômicas e aplicáveis nas condições específicas de trabalho.

Os objetivos deste trabalho foram a quantificação das exposições ocupacionais na aplicação de agrotóxicos com o pulverizador convencional do produtor em cultura de batata; a determinação das regiões do corpo dos trabalhadores mais expostas; a avaliação da eficiência de medidas de proteção individual (conjuntos AZR e Roupa Unesp) e coletiva (pulverizador Knapik e protótipo da barra manual); e a classificação das condições de trabalho quanto à segurança ocupacional das 54 recomendações de agrotóxicos registrados para o controle das principais pragas e doenças dessa cultura.

A aplicação com este equipamento é realizada por duas pessoas, que transportam manualmente a barra de bambu a 50 cm acima da extremidade das plantas da cultura. O pulverizador foi calibrado com a pressão de $2.100 \mathrm{KPa}$ e aplicou o volume de $525 \mathrm{~L} / \mathrm{ha}$. A barra de bambu foi equipada com quatorze bicos espaçados em 0,5 $\mathrm{m}$, com pontas de jato cônico, marca Magno, cor preta. A denominação das pontas de pulverização não está apresentada no presente trabalho devido ao fato destas inscrições originais estarem inelegíveis nas pontas de pulverizações utilizadas pelo produtor rural.

O protótipo da barra manual foi utilizado no lugar da barra de bambu e para isso foram realizadas apenas a substituição do equipamento do produtor e a recalibragem do pulverizador semi-estacionário. Nessa atividade, também trabalham duas pessoas 
que transportam manualmente o protótipo da barra manual, realizando a aplicação da calda na cultura. O pulverizador foi calibrado com a pressão de $280 \mathrm{KPa}$ para aplicar o volume de 385 L/ha. Foram utilizados onze bicos espaçados em $0,5 \mathrm{~m}$, com pontas de pulverização de jato plano duplo comum TwinJet ${ }^{\circledR}$ TJ60 - 11003 VS, da marca Spraying Systems Co ${ }^{\circledR}$.

A estrutura principal do protótipo da barra manual, desenvolvido e avaliado como medida de segurança coletiva, foi constituída com uma longarina de cantoneira de alumínio de 1" de largura e 3,175 mm de espessura e dois tubos de 12,7 mm. A estrutura de travamento da barra (treliça) foi feita de tubos de alumínio com 9,525 mm de diâmetro e parede de 1,588 mm.

Nas extremidades da barra, para o fechamento e a fixação da estrutura, foram utilizados dois triângulos de cantoneira (25,4 $\mathrm{mm}$ de largura e $1,588 \mathrm{~mm}$ de espessura) de 0,35 x 0,26 x 0,26 m. Nestes triângulos, foram fixadas as manetes para o transporte da barra. A altura da barra em relação ao alvo a ser pulverizado pode ser regulada mudando-se o ponto de fixação das manetes, as quais se encaixam em duas hastes verticais com vários furos.

Foi desenvolvida uma alça tipo tiracolo com regulagem de tamanho para ajustar melhor o equipamento aos trabalhadores e proporcionar maior conforto. A barra foi protegida com uma lâmina plástica (cristal 0,20 mm de espessura) contra deriva. Para fixar a lâmina plástica sobre a barra, foi desenvolvido um sistema de encaixe de extensores de tubo de alumínio com $36 \mathrm{~cm}$ de comprimento na região frontal e com 41 $\mathrm{cm}$ de comprimento na região posterior do protótipo.

O comprimento do protótipo da barra manual foi de $6 \mathrm{~m}$ e o peso, de $15,4 \mathrm{~kg}$, perfeitamente suportável por dois operadores.

O pulverizador Knapik foi constituído a partir da adaptação de um pulverizador costal manual com tanque de 20 L montado sobre uma estrutura metálica com uma roda de bicicleta. Possui uma barra frontal com altura regulável, que sustenta uma mangueira com seis bicos de pulverização espaçados em 0,5 m e sem especificação. O pulverizador foi calibrado com o regulador de pressão na posição de $75 \%$ na estrutura de regulagem da pressão para aplicar o volume de $160 \mathrm{~L} / \mathrm{h}$. Para realizar a pulverização na área do ensaio, o trabalhador empurrou o pulverizador Knapik. Assim, o caminhamento do trabalhador na área do ensaio ocorreu imediatamente após a realização da pulverização, condição mais crítica de trabalho, pois, se o trabalhador tracionasse o equipamento, provavelmente a exposição dérmica sofrida seria menor.

Nas avaliações sem medidas de segurança, as exposições foram denominadas de exposição dérmica potencial (EDP) e exposição respiratória potencial (ERP), consideradas como as quantidades máximas proporcionadas pelas condições de trabalho e que, teoricamente, poderiam atingir essas vias de exposição do trabalhador. As exposições não controladas pelas medidas de proteção individuais e coletivas avaliadas foram denominadas exposição dérmica não controlada (EDNC) e exposição respiratória não controlada (ERNC). Foram avaliadas as EDs dos trabalhadores expostos às caldas que continham o cobre de um fungicida cúprico como traçador, conforme método descrito e utilizado por Machado Neto (1997).

Os amostradores das EDs foram absorventes higiênicos femininos, para amostrar as exposições nos pés e na face, luvas de algodão para as mãos e macacão para as demais partes do corpo. As exposições foram avaliadas em períodos inferiores à uma hora de trabalho e, posteriormente, extrapoladas para um tempo de exposição efetiva (TEE) de seis horas (MACHADO NETO, 1997).

As ERPs foram amostradas e quantificadas em filtros de éster celulose da marca SKC, com porosidade de 0,8 $\mu \mathrm{m}$, utilizados em K7s de bombas de fluxo contínuo de ar, marca A. P. Buck, reguladas com a vazão de $2 \mathrm{~L} / \mathrm{min}$ (OLIVEIRA \& MACHADO NETO, 2003).

Os $\mathrm{K} 7 \mathrm{~s}$ foram posicionados na região de respiração dos trabalhadores expostos às caldas que continham o cátion manganês do sulfato de manganês utilizado como traçador (OLIVEIRA \& MACHADO NETO, 2003). As ERPs foram avaliadas por períodos de aproximadamente três horas e, posteriormente, extrapoladas para um TEE de seis horas.

As EDs e ERPs às caldas foram estimadas com os valores de cobre e de manganês quantificados nos amostradores e suas respectivas concentrações, nas caldas aplicadas. Todas as atividades foram avaliadas com 10 repetições. Os macacões, seccionados em partes (capuz, braços, tronco-frente, tronco-atrás, coxas + pernas-frente e coxas 
+ pernas-atrás), as luvas de algodão, os absorventes higiênicos femininos e os filtros coletores dos $\mathrm{K} 7 \mathrm{~s}$ foram imersos em solução de $\mathrm{HCl}$ 0,2 N para a solubilização dos cátions. Os amostradores permaneceram na solução solubilizadora por duas horas. Em seguida, as amostras foram agitadas e uma alíquota dessas soluções com cobre foi filtrada em filtro de papel qualitativo (MACHADO NETO \& MATUO, 1989). As EDs das partes do corpo do trabalhador foram avaliadas nas seguintes regiões do corpo: cabeça + pescoço, face, mãos, braços, tronco-frente, tronco-atrás, coxas + pernas -frente, coxas + pernas-atrás e pés.

Nas amostras de quantificação das ERPs com o manganês, procedeu-se apenas à retirada dos filtros de éster celulose das soluções (OLIVEIRA \& MACHADO NETO, 2003). Os cátions cobre e manganês foram quantificados nas alíquotas dessas soluções em espectrofotômetro de absorção atômica.

As condições de trabalho do espectrofotômetro de absorção atômica foram as seguintes:

a) para o cobre, lâmpada de catodo oco, corrente de 3,0 mA, comprimento de onda de $324,7 \mathrm{~nm}$, fenda de $0,5 \mathrm{~nm}$, chama de ar/ acetileno oxidante, curva-padrão de cobre preparada a partir da solução de cloreto de cobre $\left(\mathrm{CuCl}_{2}\right)$ Titrisol Merk 9987 e faixa de linearidade determinada com as concentrações de 0,125, 0,25, 0,5, 1,0 e 2,0 ppm $\left(\mathrm{R}^{2}=0,999\right)$

b) para o manganês, lâmpada de catodo oco, corrente de 5,0 mA, comprimento de onda de 279,5 nm, fenda de 0,2 nm, chama de ar/acetileno oxidante, curva-padrão de manganês preparada a partir da solução de cloreto de manganês (MnCl) Titrisol Merk 9988 e faixa de linearidade determinada com as mesmas concentrações utilizadas para o cobre $\left(\mathrm{R}^{2}=0,998\right)$. Os limites de detecção foram de 0,017 ppm e de 0,019 ppm e o limite de quantificação de 0,041 ppm e de 0,046 ppm para o cobre e para o manganês, respectivamente, calculados de acordo com Skoog et al. (1998).

As EDNCs foram avaliadas em vestimentas amostradoras usadas sob os dois conjuntos de proteção individuais e quando da utilização dos equipamentos de proteção coletivos. Foi considerada a eficiência de 95\% de controle das exposições potenciais avaliadas (LUNDEHN et al., 1992) para botas e máscara descartável com filtro de carvão ativado.
Avaliaram-se como medidas de proteção individual os conjuntos AZR e Roupa Unesp. O conjunto AZR foi composto pelas seguintes vestimentas: blusa de mangas compridas, calças compridas e touca árabe com aba frontal confeccionadas em tecido de algodão tratado com Teflon ${ }^{\circledR}$, que confere hidrorrepelência do tecido às gotas de pulverização, viseira de acetato transparente, avental de material impermeável, luvas de nitrila, botas de borracha e máscara descartável com filtro de carvão ativado.

A Roupa Unesp foi composta por uma vestimenta confeccionada em lâmina de plástico impermeável para a proteção dos braços, da parte frontal do corpo e das pernas e aberta na região posterior do corpo para proporcionar ventilação e maior conforto, chapéu de palha, viseira de acetato transparente, luvas de nitrila, botas de borracha e máscara descartável com filtro de carvão ativado.

Como medidas de proteção coletiva, foi avaliada a eficiência do protótipo da barra manual e do pulverizador Knapik na redução das exposições ocupacionais. O protótipo da barra manual utiliza os princípios de segurança por proteção da barra de pulverização com lâmina de plástico impermeável e por redução do volume de aplicação. No pulverizador Knapik, foi utilizado o princípio de proteção por distância, em que o operador fica 1,5 m distante da barra de pulverização, além da redução do volume de aplicação.

As exposições às caldas, quantificadas com os traçadores, foram utilizadas como dados substitutos, de acordo com Jensen (1984), para estimar as exposições às 54 recomendações de agrotóxicos consideradas para a aplicação nas condições avaliadas de acordo com as respectivas dosagens recomendadas e citadas no Sistema de Informações sobre Agrotóxicos (ANVISA, 2004). Esses agrotóxicos foram escolhidos por serem os mais tóxicos e/ou os mais utilizados pelos agricultores nessas condições de pulverização na cultura de batata.

A segurança das condições de trabalho avaliadas, com cada agrotóxico recomendado, foi estimada por meio do cálculo da margem de segurança (MS) com a fórmula de Severn (1984) modificada por Machado Neto (1997): $\mathrm{MS}=($ NOEL x 70) / (QAE x 10), onde: $\mathrm{MS}=$ margem de segurança; NOEL = nível de efeitos não observados (mg/kg/dia); 70 = peso corpóreo médio $(\mathrm{kg})$; 10 = fator de segurança para compensar a 
extrapolação dos valores de NOEL obtidos em animais de laboratório para o homem (BROUWER et al., 1990); e QAE = quantidade absorvível das exposições avaliadas (mg/dia), considerada como 0,1 x ED + ER. O critério utilizado para a classificação da segurança das condições de trabalho foi o seguinte: se $\mathrm{MS} \geq 1$, condição segura, exposição tolerável e risco aceitável, e se MS < 1, condição insegura, exposição intolerável e risco inaceitável, segundo Machado Neto (1997).

Para formulações compostas por dois ingredientes ativos, a segurança da condição de trabalho foi classificada com base no menor valor de MS calculado entre os ingredientes ativos.

Para as condições de trabalho inseguras, classificadas como MS $<1$, foi calculada a necessidade de controle da exposição (NCE), em termos de porcentagem da exposição avaliada, com a fórmula proposta por

\section{Resultados e Discussão}

Os resultados das exposições dérmicas às suspensões de cobre e das exposições respiratórias às suspensões de manganês, proporcionadas aos aplicadores nas condições de trabalho avaliadas, estão apresentados na Tabela 1. Verifica-se que as medidas de segurança individuais e coletivas testadas reduziram as exposições ocupa-
Machado Neto (1997): NCE $=\left(1-\mathrm{MS}_{<1}\right)$ x $100(\%)$.

Outra medida de segurança que pode ser utilizada em condições inseguras é a limitação do tempo de exposição ao tempo de trabalho seguro (TTS) calculado para a condição de trabalho (MACHADO NETO, 1997). O TTS foi calculado por meio da fórmula proposta por Machado Neto (1997): TTS = MS x TEE, onde: TTS = tempo de trabalho seguro (h), MS = margem de segurança, e TEE = tempo de exposição efetiva (h).

No cálculo do TTS, duas situações podem ocorrer: se MS $\geq 1$, o TTS será maior que o tempo de exposição considerado, possibilitando a quantificação de quão segura é a condição de trabalho em estudo, e se MS < 1, o TTS será menor que o tempo de exposição considerado e o seu cálculo possibilitará a restrição do tempo de exposição diária ao TTS, utilizando-o como medida de segurança coletiva.

Tabela 1 Exposições dérmicas, respiratórias e totais (dérmica + respiratória) sofridas pelo aplicador trabalhando com o pulverizador do produtor e com a utilização das medidas de proteção individuais e coletivas e eficiência (\%) das medidas de segurança

\begin{tabular}{lcccc}
\hline & \multicolumn{3}{c}{ Exposição (mL de calda/dia) } & Eficiência \\
\cline { 2 - 4 } \multicolumn{1}{c}{ Atividade } & Dérmica & Respiratória & Total & (\%) \\
\hline Pulverizador do produtor (EP) & $25.138,882$ & 0,117 & $25.138,999$ & - \\
$\begin{array}{l}\text { Pulverizador do produtor com Roupa } \\
\text { Unesp (ENC) }\end{array}$ & 587,190 & 0,006 & 587,196 & 97,7 \\
$\begin{array}{l}\text { Pulverizador do produtor com AZR (ENC) } \\
\text { Pulverizador Knapik (ENC) }\end{array}$ & $1.466,063$ & 0,006 & $1.466,069$ & 94,2 \\
Protótipo da barra manual (ENC) & $1.834,089$ & 0,017 & $1.834,106$ & 92,7 \\
\hline
\end{tabular}

$\mathrm{EP}=$ Exposição potencial $\quad \mathrm{ENC}=$ Exposição não controlada

O conjunto Roupa Unesp proporcionou eficiência ligeiramente maior que o conjunto AZR. Esse fato ocorreu principalmente porque o modelo do conjunto AZR utiliza- cionais dos aplicadores de agrotóxicos em cultura de batata. A exposição não controlada (ENC) pelo conjunto Roupa Unesp foi 42,8 vezes menor que a EP proporcionada pelo pulverizador do produtor, controlando-a em 97,7\%. Da mesma forma, com o conjunto AZR a ENC foi 17,2 vezes menor que a EP, controlando-a em 94,2\%. do neste trabalho não possuía proteção impermeável na região do joelho até a barra da calça, que foi a região do corpo que recebeu a maior parte da exposição. Por outro 
lado, o conjunto Roupa Unesp é totalmente impermeável, inclusive na região citada, fato que foi vantajoso no controle da EDP.

A eficiência de proteção do conjunto AZR foi similar à citada por Oliveira (2000). Esse autor verificou que o conjunto AZR controlou 93,1\% da exposição dérmica do aplicador trabalhando com o pulverizador de pistolas em citros. A eficiência de proteção do conjunto Roupa Unesp foi superior aos 78,3\% obtidos por Oliveira (2000) para o aplicador também trabalhando com o pulverizador de pistolas em citros.

Ao utilizar o protótipo da barra manual, a ENC foi 2,4 vezes menor que a EP proporcionada pelo pulverizador do produtor, controlando-a em 58,8\%. Com o pulverizador Knapik, a ENC foi 13,7 vezes menor que a EP proporcionada pelo pulverizador do produtor, controlando-a em 92,7\%. A elevada eficiência no controle da EDP obtida com o pulverizador Knapik ocorreu principalmente em decorrência do menor volume de aplicação (160 L/ha) utilizado neste equipamento. Verifica-se que esse volume é drasticamente inferior aos volumes de aplicação empregados com o pulverizador convencional do produtor (525 L/ha) e com o protótipo da barra manual (385 L/ha).

Não obstante a eficiência no controle da EDP obtida com o pulverizador Knapik, o volume de aplicação empregado para a realização deste ensaio pode não ser suficiente para o tratamento fitossanitário da cultura da batata. Essa condição foi empregada devido à dificuldade de locomoção do pulverizador na área montanhosa onde se instalou o ensaio. Essa dificuldade de locomoção ocorreu devido ao fato do dispositivo regulador de pressão do pulverizador ser acoplado à roda do equipamento. Nas condições montanhosas do ensaio, essa regulagem foi a que proporcionou maior volume de aplicação possível, pois, com as regulagens para obtenção de maiores pressões, ocorreu o patinamento da roda do pulverizador no solo, impossibilitado o trabalho nessas condições.

Na Tabela 2, estão apresentadas as distribuições percentuais das EDPs e das EDNCs recebidas nas regiões do corpo do trabalhador nas atividades estudadas. Verifica-se que a EDP recebida pelo aplicador com o pulverizador do produtor se concentrou nas coxas + pernas-frente, nos pés e nas coxas + pernas-atrás, que receberam, respectivamente, $39,4 \%, 38,9 \%$ e $21,1 \%$ da EDP total, ou seja, 99,4\% da exposição dérmica proporcionada pela atividade. As regiões do corpo mais expostas, em ordem decrescente, foram: coxas + pernas-frente, pés, coxas + pernas-atrás, mãos, braços, tronco-atrás, tronco-frente, cabeça + pescoço e face.

Tabela 2 Distribuição das exposições dérmicas em $\mathrm{mL}$ de calda por dia e em porcentagem nas diversas regiões do corpo do trabalhador e exposição dérmica em $\mathrm{mL}$ de calda por dia aplicando agrotóxicos com os pulverizadores do produtor, o protótipo da barra manual, o Knapik, o pulverizador do produtor com AZR e com a Roupa Unesp em cultura de batata

\begin{tabular}{|c|c|c|c|c|c|c|c|c|c|c|}
\hline \multirow{2}{*}{ Partes do corpo } & \multicolumn{2}{|c|}{$\begin{array}{l}\text { Pulverizador do } \\
\text { produtor }\end{array}$} & \multicolumn{2}{|c|}{ Roupa Unesp } & \multicolumn{2}{|c|}{$A Z R$} & \multicolumn{2}{|c|}{ Knapik } & \multicolumn{2}{|c|}{ Protótipo } \\
\hline & $m L / d i a$ & $\%$ & $m L / d i a$ & $\%$ & $m L / d i a$ & $\%$ & $m L / d i a$ & $\%$ & $m L / d i a$ & $\%$ \\
\hline Cabeça + pescoço & 2,5 & 0,01 & 0,1 & 0,02 & 12,8 & 0,87 & 4,6 & 0,25 & 4,1 & 0,04 \\
\hline Face & 0,0 & 0,00 & 0,5 & 0,09 & 0,1 & 0,01 & 8,4 & 0,46 & 2,1 & 0,02 \\
\hline Mãos & 45,3 & 0,18 & 0,2 & 0,03 & 14,4 & 0,98 & 54,5 & 2,97 & 23,9 & 0,23 \\
\hline Braços & 37,7 & 0,15 & 0,2 & 0,04 & 12,3 & 0,84 & 42,9 & 2,34 & 20,7 & 0,20 \\
\hline Tronco-frente & 15,1 & 0,06 & 0,1 & 0,02 & 99,3 & 6,77 & 22,9 & 1,25 & 37,3 & 0,36 \\
\hline Tronco-atrás & 25,4 & 0,10 & 0,1 & 0,02 & 20,8 & 1,42 & 10,8 & 0,59 & 13,5 & 0,13 \\
\hline Coxas + pernas-frente & $9.912,5$ & 39,43 & 47,5 & 8,09 & 752,1 & 51,30 & 1025,8 & 55,93 & $4.476,6$ & 43,17 \\
\hline Coxas + pernas-atrás & $5.312,9$ & 21,13 & 61,5 & 10,47 & 290,9 & 19,84 & 135,9 & 7,41 & $3.418,9$ & 32,97 \\
\hline Pés & $9.787,6$ & 38,93 & 476,9 & 81,21 & 263,5 & 17,97 & 528,2 & 28,80 & $2.372,6$ & 22,88 \\
\hline $\begin{array}{l}\text { Exposição dérmica } \\
\text { (mL/dia) }\end{array}$ & \multicolumn{2}{|c|}{$25.138,9$} & \multicolumn{2}{|c|}{587,2} & \multicolumn{2}{|c|}{$1.466,1$} & \multicolumn{2}{|c|}{$1.834,1$} & \multicolumn{2}{|c|}{$10.369,8$} \\
\hline
\end{tabular}


Para o aplicador com o pulverizador do produtor que utilizou o conjunto Roupa Unesp como medida de proteção individual, a distribuição da EDNC também se concentrou nas partes inferiores do corpo, porém com inversão na ordem de grandeza, sendo as partes mais expostas pés, coxas + pernas-atrás e coxas + pernasfrente, recebendo, respectivamente, $81,2 \%$, $10,5 \%$ e $8,1 \%$ da exposição total $(99,8 \%)$. As regiões do corpo mais expostas nesta atividade, em ordem decrescente, foram pés, coxas + pernas-atrás, coxas + pernasfrente, face, braços, mãos, tronco-atrás, tronco-frente e cabeça + pescoço. A maior exposição dos pés foi incrementada pelo escorrimento da calda de pulverização que atingiu as coxas + pernas-frente, pois a Roupa Unesp é impermeável nessas regiões do corpo. As coxas + pernas-atrás receberam maior exposição que as coxas + pernas-frente devido à abertura que a Roupa Unesp tem na região posterior do corpo.

Para o aplicador que utilizou o conjunto AZR como medida de proteção individual, a EDNC também se concentrou nas três partes inferiores do corpo do aplicador: coxas + pernas-frente, coxas + pernas-atrás e pés, que receberam uma exposição dérmica de $51,3 \%, 19,8 \%$ e $18,0 \%$ da ED total, respectivamente. As regiões do corpo mais expostas, em ordem decrescente, foram: coxas + pernas-frente, coxas + pernas-atrás, pés, tronco-frente, tronco-atrás, mãos, cabeça + pescoço, braços e face. Observa-se que as coxas + pernas-frente foram as regiões mais expostas. Tal fato ocorreu devido ao conjunto AZR utilizado nesta avaliação não possuir a proteção impermeável para esta região do corpo.

A utilização do conjunto AZR com proteção impermeável na frente das pernas, conforme indicação do fabricante do equipamento, poderia ter proporcionado maior redução na exposição dérmica nesta região do corpo do trabalhador. O conjunto AZR sem proteção impermeável foi utilizado exatamente para que se pudesse avaliar a diferença entre um material hidrorrepelente e um impermeável (Roupa Unesp) em condições de alta exposição ocupacional às caldas de agrotóxicos. Comparado com a Roupa Unesp, verifica-se que, no AZR, ocorre na região do tronco-frente uma exposição de $6,8 \%$ da exposição total, fato não observado na Roupa Unesp devido à sua impermeabilidade.
Com o protótipo da barra manual, utilizado como medida de proteção coletiva, a distribuição percentual da exposição dérmica do aplicador ocorreu principalmente nas coxas + pernas-frente, nas coxas + pernas-atrás e nos pés, que receberam $43,2 \%$, 33\% e 22,9\% da exposição total, respectivamente. As regiões mais expostas, em ordem decrescente, foram: coxas + pernas-frente, coxas + pernas-atrás, pés, tronco-frente, mãos, braços, tronco-atrás, cabeça + pescoço e face. Verifica-se que 99,1\% da exposição dérmica ocorreram nas três regiões do corpo anteriormente citadas, distribuição semelhante à EDP proporcionada pelo pulverizador do produtor sem nenhuma medida de segurança.

A distribuição percentual da exposição dérmica sofrida pelo aplicador, utilizando o pulverizador Knapik como medida de proteção coletiva, ocorreu principalmente nas coxas + pernas-frente, nos pés e nas coxas + pernas-atrás, com 55,9\%, 28,8\% e 7,4\%, respectivamente. As regiões mais expostas, em ordem decrescente, foram: coxas + pernas-frente, pés, coxas + pernas-atrás, mãos, braços, tronco-frente, tronco-atrás, face e cabeça + pescoço. Observa-se que 92,1\% da exposição dérmica também ocorreram nas três regiões do corpo citadas, distribuição semelhante à EDP proporcionada pelo pulverizador do produtor sem nenhuma medida de segurança. As regiões do corpo do trabalhador mais expostas nas aplicações de agrotóxicos estudadas na cultura da batata foram as coxas + pernas-frente, os pés e as coxas + pernas-atrás.

Essa característica de concentração da exposição dérmica nessas partes do corpo do aplicador de agrotóxicos na cultura de batata indica a possibilidade de recomendar equipamentos de proteção apenas para essas regiões do corpo, proporcionando conforto e eficiência no controle da exposição sofrida pelo trabalhador. Assim, podem-se recomendar EPIs impermeáveis que protejam os membros inferiores dos aplicadores, pois apenas a hidrorrepelência não foi suficiente para a proteção devido à elevada quantidade de calda que atinge essas regiões do corpo do trabalhador. A eficiência da impermeabilização pode ser observada nas coxas + pernas-frente, comparando-se as EDNCs pelo conjunto AZR (752 mL/dia) e a Roupa Unesp (47,5 mL/dia). Verifica-se que, com a Roupa Unesp (impermeável), a EDNC foi 93,7\% menor que com o conjunto AZR (hidrorrepelente). 
Os valores de margem da segurança (MS) e do tempo de trabalho seguro (TTS), calculados nas condições de trabalho com os três pulverizadores e com os dois con- juntos de proteção individual para as recomendações de agrotóxicos registrados para o controle das principais pragas e doenças da cultura da batata estão apresentados na Tabela 3.

Tabela 3 Margem de segurança (MS) e tempo de trabalho seguro (TTS) calculados para as recomendações de agrotóxicos registrados para o controle das principais pragas e doenças da cultura da batata

\begin{tabular}{|c|c|c|c|c|c|c|c|c|c|c|}
\hline \multirow{2}{*}{ Ingrediente ativo } & \multicolumn{2}{|c|}{ Pulv. produtor } & \multicolumn{2}{|c|}{ Roupa Unesp } & \multicolumn{2}{|c|}{$A Z R$} & \multicolumn{2}{|c|}{ Knapik } & \multicolumn{2}{|c|}{ Protótipo } \\
\hline & MS & $\pi \mathrm{S}$ & MS & $\pi \mathrm{S}$ & MS & $T T S$ & MS & $\pi \mathrm{S}$ & MS & TTS \\
\hline acephate & 0,0003 & 0,0015 & 0,022 & 0,1320 & 0,0088 & 0,0529 & 0,0064 & 0,0383 & 0,0010 & 0,0061 \\
\hline acetamiprid & 0,1011 & 0,6063 & 8,856 & 53,1383 & 3,5471 & 21,2829 & 0,7829 & 4,6973 & 0,3022 & 1,8132 \\
\hline alpha-cipermetrin & 0,3015 & 1,8093 & 26,428 & 158,5715 & 10,5851 & 63,5108 & 7,6658 & 45,9948 & 1,2297 & 7,3783 \\
\hline azoxystrobin & 0,0842 & 0,5053 & 7,380 & 44,2819 & 2,9560 & 17,7357 & 0,6524 & 3,9144 & 0,2518 & 1,5110 \\
\hline benalaxyl + mancozeb & 0,0002 & 0,0012 & 0,018 & 0,1090 & 0,0073 & 0,0437 & 0,0016 & 0,0096 & 0,0006 & 0,0037 \\
\hline beta-cyfluthrin & 0,0808 & 0,4850 & 7,085 & 42,5107 & 2,8377 & 17,0263 & 0,6263 & 3,7579 & 0,2418 & 1,4505 \\
\hline bromuconazole & 0,0090 & 0,0539 & 0,787 & 4,7234 & 0,3153 & 1,8918 & 0,0696 & 0,4175 & 0,0269 & 0,1612 \\
\hline captan & 0,0674 & 0,4042 & 5,904 & 35,4256 & 2,3648 & 14,1886 & 0,5219 & 3,1316 & 0,2015 & 1,2088 \\
\hline carbaryl & 0,0161 & 0,0966 & 1,411 & 8,4677 & 0,5652 & 3,3915 & 0,4094 & 2,4561 & 0,0657 & 0,3940 \\
\hline cartap & 0,0103 & 0,0616 & 0,899 & 5,3982 & 0,3603 & 2,1621 & 0,2610 & 1,5658 & 0,0419 & 0,2512 \\
\hline chlorfenapyr & 0,0079 & 0,0472 & 0,688 & 4,1330 & 0,2759 & 1,6553 & 0,0609 & 0,3653 & 0,0235 & 0,1410 \\
\hline chlorothalonil & 0,0007 & 0,0040 & 0,059 & 0,3543 & 0,0236 & 0,1419 & 0,0052 & 0,0313 & 0,0020 & 0,0121 \\
\hline chlorothalonil + propamocarb & 0,0009 & 0,0054 & 0,078 & 0,4723 & $0 w, 0315$ & 0,1892 & 0,0070 & 0,0418 & 0,0027 & 0,0161 \\
\hline chlorpyrifos & 0,0000 & 0,0002 & 0,002 & 0,0133 & 0,0009 & 0,0053 & 0,0002 & 0,0012 & 0,0001 & 0,0005 \\
\hline cymoxanil + famoxadone & 0,0153 & 0,0921 & 1,344 & 8,0692 & 0,5386 & 3,2318 & 0,1189 & 0,7133 & 0,0459 & 0,2753 \\
\hline cymoxanil + mancozeb & 0,0006 & 0,0036 & 0,052 & 0,3163 & 0,0211 & 0,1267 & 0,0153 & 0,0917 & 0,0025 & 0,0147 \\
\hline cymoxanil + maneb & 0,0173 & 0,1036 & 1,513 & 9,0778 & 0,6060 & 3,6358 & 0,1337 & 0,8025 & 0,0516 & 0,3098 \\
\hline cyprodinil & 0,0097 & 0,0582 & 0,850 & 5,1013 & 0,3405 & 2,0432 & 0,0752 & 0,4509 & 0,0290 & 0,1741 \\
\hline cyromazine & 0,0135 & 0,0808 & 1,180 & 7,0851 & 0,4730 & 2,8377 & 0,1044 & 0,6263 & 0,0403 & 0,2418 \\
\hline deltamethrin & 0,1283 & 0,7699 & 11,242 & 67,4773 & 4,5043 & 27,0259 & 3,2620 & 19,5722 & 0,5233 & 3,1397 \\
\hline deltamethrin + triazophos & 0,0001 & 0,0008 & 0,011 & 0,0675 & 0,0045 & 0,0270 & 0,0010 & 0,0060 & 0,0004 & 0,0023 \\
\hline difeconazole & 0,0090 & 0,0539 & 0,787 & 4,7234 & 0,3153 & 1,8918 & 0,0696 & 0,4175 & 0,0269 & 0,1612 \\
\hline dimethoate & 0,0004 & 0,0026 & 0,037 & 0,2249 & 0,0150 & 0,0901 & 0,0109 & 0,0652 & 0,0017 & 0,0105 \\
\hline dimethomorph & 0,0101 & 0,0606 & 0,885 & 5,3138 & 0,3547 & 2,1283 & 0,0783 & 0,4697 & 0,0302 & 0,1813 \\
\hline fenamidone & 0,0027 & 0,0162 & 0,236 & 1,4170 & 0,0946 & 0,5675 & 0,0209 & 0,1253 & 0,0081 & 0,0484 \\
\hline fenitrothion & 0,0001 & 0,0005 & 0,007 & 0,0472 & 0,0032 & 0,0189 & 0,0007 & 0,0042 & 0,0003 & 0,0016 \\
\hline fentin acetate & 0,0012 & 0,0072 & 0,105 & 0,6326 & 0,0422 & 0,2534 & 0,0093 & 0,0559 & 0,0036 & 0,0216 \\
\hline fentin hydroxide & 0,0004 & 0,0026 & 0,037 & 0,2249 & 0,0150 & 0,0901 & 0,0109 & 0,0652 & 0,0017 & 0,0105 \\
\hline fipronil & 0,0001 & 0,0005 & 0,007 & 0,0443 & 0,0030 & 0,0177 & 0,0007 & 0,0039 & 0,0003 & 0,0015 \\
\hline fluazinam & 0,0005 & 0,0032 & 0,047 & 0,2834 & 0,0189 & 0,1135 & 0,0042 & 0,0251 & 0,0016 & 0,0097 \\
\hline imidacloprid & 0,0220 & 0,1320 & 1,927 & 11,5675 & 0,7722 & 4,6330 & 0,5592 & 3,3552 & 0,0897 & 0,5382 \\
\hline
\end{tabular}


Tabela 3 Margem de segurança (MS) e tempo de trabalho seguro (TTS) calculados para as recomendações de agrotóxicos registrados para o controle das principais pragas e doenças da cultura da batata

\begin{tabular}{|c|c|c|c|c|c|c|c|c|c|c|}
\hline \multirow{2}{*}{$\begin{array}{l}(\ldots) \\
\quad \text { Ingrediente ativo }\end{array}$} & \multicolumn{2}{|c|}{ Pulv. produtor } & \multicolumn{2}{|c|}{ Roupa Unesp } & \multicolumn{2}{|c|}{$A Z R$} & \multicolumn{2}{|c|}{ Knapik } & \multicolumn{2}{|c|}{ Protótipo } \\
\hline & MS & TTS & MS & $\pi \mathrm{S}$ & MS & $\pi \mathrm{S}$ & MS & TTS & MS & $\pi T S$ \\
\hline iprodione & 0,0068 & 0,0411 & 0,599 & 3,5988 & 0,2402 & 1,4414 & 0,1740 & 1,0439 & 0,0279 & 0,1675 \\
\hline iprovalicarb + propineb & 0,0002 & 0,0013 & 0,019 & 0,1156 & 0,0077 & 0,0463 & 0,0017 & 0,0102 & 0,0007 & 0,0039 \\
\hline kasugamycin & 0,9624 & 5,7743 & 84,346 & 506,0794 & 33,7823 & 202,694 & 24,4653 & 146,792 & 3,9246 & 23,5478 \\
\hline kresoxim-methyl & 0,1213 & 0,7276 & 10,627 & 63,7660 & 4,2566 & 25,5395 & 0,9395 & 5,6368 & 0,3626 & 2,1758 \\
\hline lambda-cyhalothrin & 0,0026 & 0,0154 & 0,224 & 1,3495 & 0,0901 & 0,5405 & 0,0652 & 0,3914 & 0,0105 & 0,0628 \\
\hline mancozeb & 0,0002 & 0,0010 & 0,014 & 0,0886 & 0,0059 & 0,0355 & 0,0013 & 0,0078 & 0,0005 & 0,0030 \\
\hline mancozeb + metalaxyl & 0,0002 & 0,0012 & 0,018 & 0,1063 & 0,0071 & 0,0426 & 0,0016 & 0,0094 & 0,0006 & 0,0036 \\
\hline metconazole & 0,0359 & 0,2156 & 3,148 & 18,8936 & 1,2612 & 7,5672 & 0,2784 & 1,6702 & 0,1074 & 0,6447 \\
\hline methamidophos & 0,0001 & 0,0008 & 0,011 & 0,0675 & 0,0045 & 0,0270 & 0,0033 & 0,0196 & 0,0005 & 0,0031 \\
\hline methomyl & 0,0075 & 0,0448 & 0,653 & 3,9231 & 0,2619 & 1,5713 & 0,1897 & 1,1379 & 0,0304 & 0,1825 \\
\hline monocrotophos & 0,0000 & 0,0000 & 0,001 & 0,0040 & 0,0003 & 0,0016 & 0,0002 & 0,0012 & 0,0000 & 0,0002 \\
\hline myclobutanil & 0,0047 & 0,0280 & 0,409 & 2,4562 & 0,1640 & 0,9837 & 0,0362 & 0,2171 & 0,0140 & 0,0838 \\
\hline parathion-methyl & 0,0000 & 0,0003 & 0,004 & 0,0225 & 0,0015 & 0,0090 & 0,0011 & 0,0065 & 0,0002 & 0,0010 \\
\hline pirimicarb & 0,0021 & 0,0123 & 0,179 & 1,0796 & 0,0721 & 0,4324 & 0,0522 & 0,3132 & 0,0084 & 0,0502 \\
\hline procymidone & 0,0086 & 0,0513 & 0,749 & 4,4985 & 0,3003 & 1,8017 & 0,2175 & 1,3048 & 0,0349 & 0,2093 \\
\hline propamocarb & 0,0047 & 0,0280 & 0,408 & 2,4533 & 0,1638 & 0,9826 & 0,0361 & 0,2169 & 0,0140 & 0,0837 \\
\hline propineb & 0,0002 & 0,0010 & 0,014 & 0,0843 & 0,0056 & 0,0338 & 0,0012 & 0,0075 & 0,0005 & 0,0029 \\
\hline prothiofos & 0,0001 & 0,0003 & 0,004 & 0,0270 & 0,0018 & 0,0108 & 0,0013 & 0,0078 & 0,0002 & 0,0013 \\
\hline pyrimethanil & 0,0254 & 0,1527 & 2,230 & 13,3830 & 0,8934 & 5,3601 & 0,1972 & 1,1830 & 0,0761 & 0,4567 \\
\hline spinosad & 0,0080 & 0,0481 & 0,702 & 4,2173 & 0,2815 & 1,6891 & 0,0621 & 0,3728 & 0,0240 & 0,1439 \\
\hline tebuconazole & 0,0051 & 0,0303 & 0,442 & 2,6569 & 0,1774 & 1,0641 & 0,0391 & 0,2349 & 0,0151 & 0,0907 \\
\hline thiacloprid & 0,0168 & 0,1011 & 1,476 & 8,8564 & 0,5912 & 3,5471 & 0,1305 & 0,7829 & 0,0504 & 0,3022 \\
\hline triazophos & 0,0001 & 0,0005 & 0,007 & 0,0443 & 0,0030 & 0,0177 & 0,0007 & 0,0039 & 0,0003 & 0,0015 \\
\hline
\end{tabular}

Com a utilização do pulverizador do produtor sem nenhuma medida de segurança, todas as recomendações de agrotóxicos consideradas foram classificadas como inseguras para o trabalhador (MS < 1). Apenas as recomendações de alpha-cipermetrin e de kasugamycin foram seguras (MS $\geq 1$ ) com a utilização das quatro medidas de segurança estudadas. A recomendação de deltamethrin foi segura com a utilização da Roupa Unesp, do AZR e com o pulverizador Knapik. As recomendações de acetamiprid, azoxystrobin, beta-cyfluthrin, captan, kresoxim-methyl e metconazole foram seguras apenas com a utilização da Roupa Unesp e com o conjunto AZR. As recomendações de carbaryl, cymoxa- nil + famoxadone, cymoxanil + maneb, cyromazine, imidacloprid, pyrimethanil e thiacloprid foram seguras somente com a utilização da Roupa Unesp.

Verifica-se que o tempo de trabalho seguro só é viável para um tempo de, pelo menos, três horas de trabalho. Portanto, ao calcular o TTS para o aplicador com o pulverizador do produtor, verificou-se que apenas na aplicação de kasugamycin o TTS foi superior a três horas de trabalho. Com o uso da Roupa Unesp, foram superiores a três horas os TTSs calculados para as recomendações de bromuconazole, cartap, cholrfenapyr, cyprodinil, difeconzole, di- 
methomorph, iprodione, methomyl, procymidone e spinosad.

Com a utilização do protótipo da barra manual, foi calculado um TTS superior a três horas apenas na recomendação de del-

\section{Conclusões}

Com os resultados obtidos, pôde-se concluir que a maior exposição ocupacional ocorreu na via dérmica, que se destacou como a de maior importância para estes trabalhadores; o trabalhador sofreu maior exposição ocupacional com a utilização do pulverizador do produtor; o pulverizador Knapik foi a medida de segurança coletiva mais eficiente no controle da exposição do trabalhador; entre os dois conjuntos de proteção individual, a Roupa Unesp foi a medida de segurança individual mais eficiente no controle da exposição tamethrin. Com a utilização do pulverizador Knapik, foram calculados TTSs superiores a três horas para as recomendações de acetamiprid, azoxystrobin, beta-cyfluthrin, captan, deltamethrin, imidacloprid e kresoxim-methyl.

\section{Agradecimentos}

do trabalhador; os membros inferiores do trabalhador foram, das regiões do corpo, os mais expostos às caldas de pulverização; sem a utilização das medidas de segurança avaliadas, todas as 54 recomendações de agrotóxico para a cultura da batata foram classificadas como inseguras $(\mathrm{MS}<1)$ para o trabalhador; todavia, com a utilização do protótipo de barra manual protegida, apenas duas tornaram-se seguras (MS $\geq 1$ ); com o Knapik somente três; nove com o AZR; e dezesseis com a Roupa Unesp.

Os autores agradecem à Fundação de Amparo à Pesquisa do Estado de São Paulo - FAPESP, pelo auxílio financeiro concedido para a realização deste trabalho.

\section{Referências Bibliográficas}

ANVISA (Agência Nacional de Vigilância Sanitária). Sistema de informações sobre agrotóxicos. Brasília. Disponível em: http:// www4.anvisa.gov.br/AGROSIA/asp/frm dados_ingrediente.asp. Acesso em: 24 mar. 2004.

BROUWER, D. H. et al. Respiratory exposure to field-strength dusts in greenhouses during application and after re-entry. Annual report 1990, TNO Health Research, p. 183-184, 1990.

JENSEN, J. K. The assumptions used for exposure assessments. In: SIEWIERSKI, M. (ed.). Determination and assessment of pesticide exposure. New York: Elsevier, 1984. p. 147-152.

LUNDEHN, J. et al. Uniform principles for safeguarding the health of applicators of plant protection products (Uniform principles for operator protection). Berlin: Kommissionsverlag Paul Parey, 1992. 90p.

MACHADO NETO, J. G. Estimativas do tempo de trabalho seguro e da necessidade de controle da exposição dos aplicadores de agrotóxicos. 1997. 83f. Tese (Livre-Docência) - Faculdade de Ciências Agrárias e Veterinárias, Universidade Estadual Paulista.

MACHADO NETO, J. G.; \& MATUO, T. Avaliação de um amostrador para o estudo da exposição dérmica potencial de aplicadores de defensivos agrícolas. Ciência Agronômica, v. 4, n. 2, p. 21-22. 1989.

OLIVEIRA, M. L. Segurança no trabalho de aplicação de agrotóxicos com turboatomizador e pulverizador de pistolas em citros. 2000. 99f. Dissertação (Mestrado) - Faculdade de Ciências Agrárias e Veterinárias, Universidade Estadual Paulista.

OLIVEIRA, M. L.; \& MACHADO NETO, J. G. Use of manganese as tracer in the determination of respiratory exposure and relative importance of exposure routes in the safety of pesticide applicators in citrus orchards. Bul. Environ. Contam. Toxicol., v. 70, p. 415-421. 2003.

SEVERN, D. J. Use of exposure data for risk assessment. In: SIEWIERSKI M. (ed.). De- 
termination and assessment of pesticide exposure. New York: Elsevier, 1984. p. 13-19.

SKOOG, D. A.; HOLLER, F. J.; \& NIEMAN, T. A. Principles of Instrumental Analysis, $5^{\text {th }}$ ed. Philadelphia: Saunders College, 1998.
SINDAG (Sindicato da Indústria de Produtos para Defesa Agrícola). Vendas de defensivos agrícolas por cultura de destinação e classes. São Paulo. Disponível em: http:// www.sindag.com.br/db/arqs/CULTURAS9700.xls. Acesso em: março de 2004. 